\title{
Novel prognostic marker PRMT1 regulates cell growth via downregulation of CDKN1A in HCC
}

\author{
Jea-Woon Ryu ${ }^{1, *}$, Seon-Kyu Kim ${ }^{1, *}$, Mi-Young Son ${ }^{1,2}$, Su-Jin Jeon ${ }^{1,2}$, Jung-Hwa Oh ${ }^{3}$, \\ Jung Hwa Lim ${ }^{1}$, Sunwha Cho ${ }^{1}$, Cho-Rok Jung ${ }^{1,2}$, Ryuji Hamamoto ${ }^{4}$, Dae-Soo Kim ${ }^{1,2}$ \\ and Hyun-Soo Cho ${ }^{1}$ \\ ${ }^{1}$ Korea Research Institute of Bioscience and Biotechnology, Daejeon, Republic of Korea \\ ${ }^{2}$ Department of Functional Genomics, Korea University of Science and Technology, Daejeon, Republic of Korea \\ ${ }^{3}$ Korea Institute of Toxicology(KIT), Daejeon, Republic of Korea \\ ${ }^{4}$ Division of Molecular Modification and Cancer Biology, National Cancer Center, Tokyo, Japan \\ *Authors share co-first authorship \\ Correspondence to: Hyun-Soo Cho, email: chohs@kribb.re.kr \\ Dae-Soo Kim, email: kds2465@kribb.re.kr \\ Keywords: HCC; PRMTl; prognostic marker \\ Received: April 14, $2017 \quad$ Accepted: December 05, $2017 \quad$ Published: December 14, 2017 \\ Copyright: Ryu et al. This is an open-access article distributed under the terms of the Creative Commons Attribution License 3.0 \\ (CC BY 3.0), which permits unrestricted use, distribution, and reproduction in any medium, provided the original author and source \\ are credited.
}

\section{ABSTRACT}

Hepatocellular carcinoma (HCC) is a major type of liver cancer caused by the hepatitis B and C viruses, alcohol and exposure to aflatoxin. For HCC treatment, anticancer drugs have been widely used, but drug resistance in advanced HCC is an important problem, resulting in a continuous need for novel therapeutic targets. Therefore, in this study, we established a screening pipeline based on RNA-seq to screen novel therapeutic/prognostic targets in HCC and identified PRMT1 (Protein Arginine Methyltransferase 1). In the prognostic analysis, the overexpression of PRMT1 was clearly associated with poor prognosis in a number of HCC patient cohorts. Moreover, after PRMT1 knockdown, HCC cell lines exhibited cell growth and spheroid formation suppression, an increase in Sub-G1 cells by FACS analysis, and enrichment of the cell cycle pathway via functional enrichment analysis. With these results, we demonstrated that PRMT1 could be a novel prognostic marker and therapeutic target for HCC therapy.

\section{INTRODUCTION}

Liver cancer is the second leading cause of death in cancer patients, and hepatocellular carcinoma (HCC) is the most common form of primary liver cancer. Chronic hepatitis $\mathrm{B}$ and $\mathrm{C}$ virus (HBV and $\mathrm{HCV}$, respectively) infections are important causes of HCC; alcohol, exposure to aflatoxin and diabetes are other factors in HCC pathogenesis [1,2]. Liver transplantation is the most efficient therapy to cure HCC. Chemotherapy and surgical removal are also recognized as alternative methods for HCC treatment. For example, sorafenib is a multi-kinase inhibitor for reducing HCC proliferation and angiogenesis via inhibiting Raf-1, B-Raf, VEGFRs and PDGFR [3].
However, sorafenib resistance in advanced HCC is considered a global issue. Thus, the development of novel therapeutic targets is needed [4].

HCC progression involves several deregulated signaling pathways. Among them, epigenetic regulation involving DNA methylation, histone modification (methylation/acetylation) and non-coding RNAs have been studied for drug development for HCC treatment [57]. The histone methylation status is highly correlated with the clinicopathological implications of HCC, and high levels of histone $\mathrm{H} 3$ lysine 4 trimethylation (H3K4me3) and $\mathrm{H} 3 \mathrm{~K} 27 \mathrm{me} 3$ are correlated with poor prognosis in $\mathrm{HCC}$ $[8,9]$. Several histone methyltransferases/demethylases are associated with the regulation of histone methylation 
in $\mathrm{HCC}$ [5]. EZH2, which trimethylates H3K27 for transcriptional silencing, is overexpressed and associated with HCC progression and aggressive behavior [10, 11]. The histone demethylase KDM1A is also highly expressed and is an independent predictor of worse prognoses in the 5-year overall survival of HCC [12]. However, other histone methyltransferases/demethylases have not been well-studied with respect to hepatocellular malignancy or as prognostic markers in $\mathrm{HCC}$.

PRMT1 is a histone arginine methyltransferase that mainly catalyzes H3R4me and me2 to activate gene expression $[13,14]$. Several papers have reported that PRMT1 expression is elevated in various types of cancer, including lung cancer, bladder cancer, breast cancer and acute myeloid leukemia, and that PRMT1 knockdown induces the suppression of cancer cell growth and metastasis [14-16]. In HCC, PRMT1 expression is targeted by miR-503, which is related to HCC metastasis regulation. However, the functions and prognostic features of PRMT1 are not yet fully understood [17]. Therefore, in this study, we identified PRMT1 overexpression using 50 normal liver and 371 HCC samples in TCGA and revealed its prognostic value in HCC using multiple patient cohorts. Thus, our findings indicate that PRMT1 may be a novel prognostic marker for HCC and that targeting PRMT1mediated proliferation may be an effective HCC therapy.

\section{RESULTS}

\section{Outline of experimental steps for screening for novel prognostic markers in $\mathrm{HCC}$}

Figure 1A shows a flowchart with the following steps for constructing a screening system to identify novel prognostic markers in HCC: 1) Collect RNAseq data (Normal/Cancer) TCGA portal; 2) Analyze expression levels using an in silico panel including histone methyltransferases/demethylases; 3) Test the possibility of a prognostic marker by Kaplan-Meier plot and log-rank test $(P<0.05)$; and 4) Analyze the function/pathway. Using this screening pipeline, we identified PRMT1 as a

A

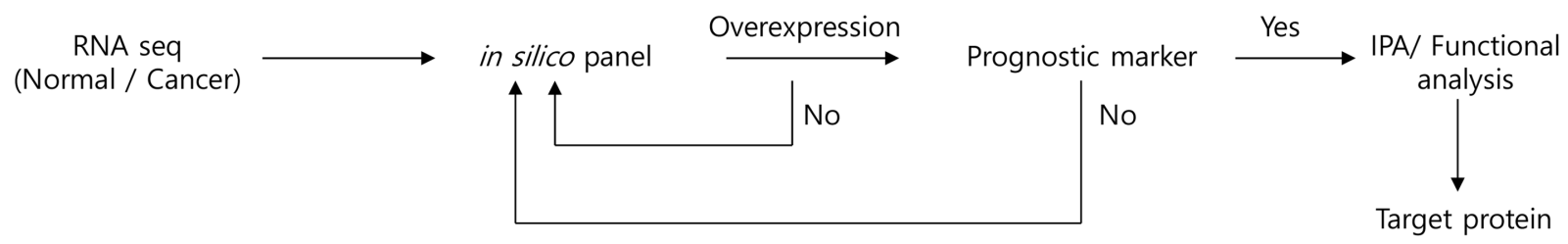

B

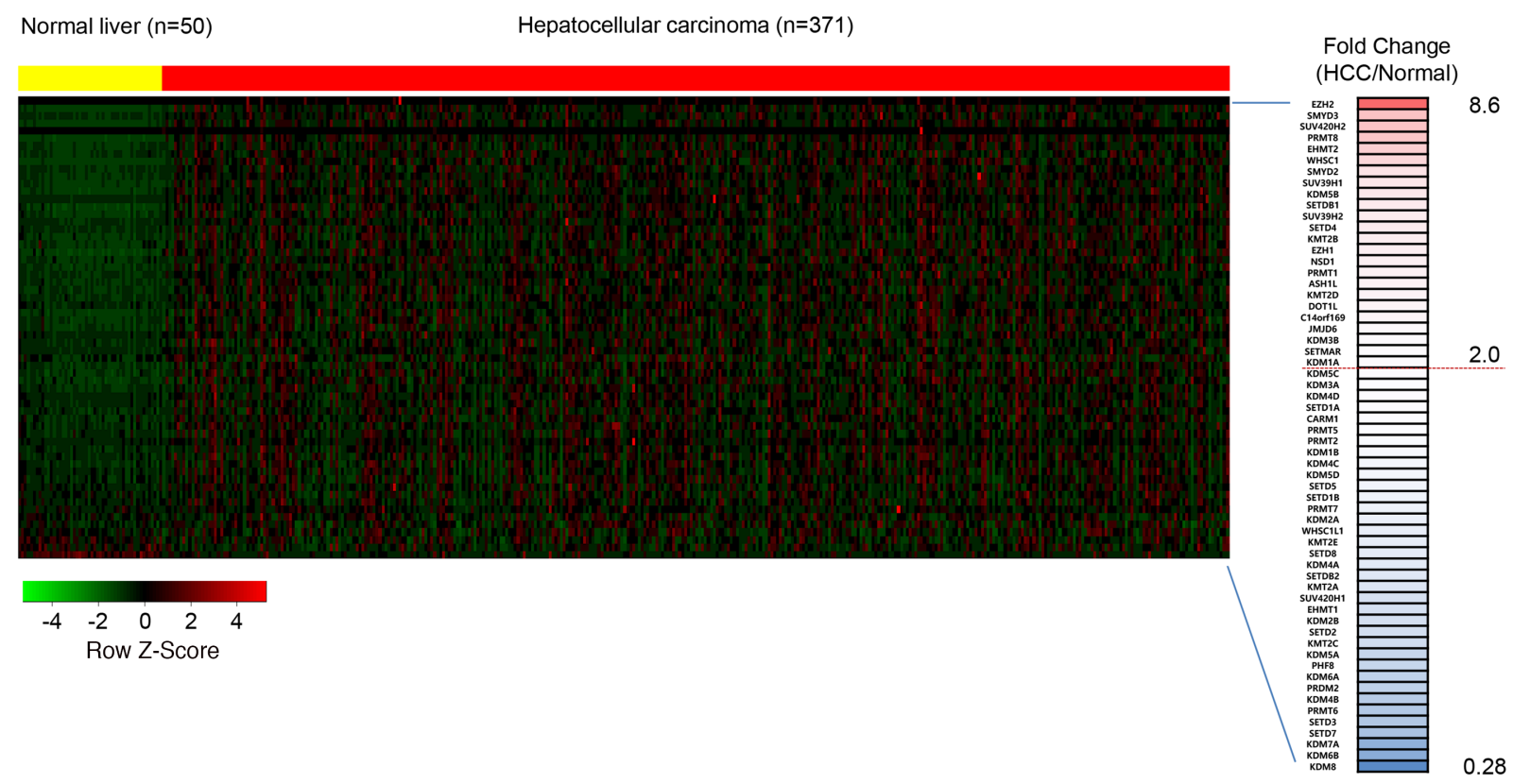

Figure 1: (A) A workflow for screening the prognostic/therapeutic marker in HCC. (B) A heat map of gene expression-related histone methylation/demethylation in the in silico histone methyltransferases/demethylases panel sorted by fold change of HCC/normal FPKM value. In the heat map, yellow indicates normal liver samples while red indicates HCC samples in TCGA. The right hand column shows gene name and fold change. The threshold is set at 2.0 -fold change. 
novel prognostic marker in HCC and demonstrated it as a therapeutic target for HCC treatment.

\section{Overexpression of histone methyltransferases and demethylases in $\mathrm{HCC}$}

From the TCGA data portal, we obtained RNAseq results from 50 normal livers and $371 \mathrm{HCC}$ samples to assess HCC-related histone methyltransferases and demethylases. We used these data to construct an in silico gene panel with 60 histone methyltransferases/demethylases (Figure 1B) for the analysis. We observed various histone methyltransferase/demethylase expression patterns in HCC compared with normal liver tissues (Figure 1B). Specifically, the fold changes (FCs) ranged from 0.28 (KDM8) to 8.6 (EZH2), and 21 histone methyltransferases and demethylases were more than 2 FC overexpressed in HCC compared with normal livers (Figure 2). Of note, EZH2 (8.6 FC) and KDM1A (2 FC) are involved in tumor proliferation and metastasis and are recognized as prognostic/diagnostic biomarkers for $\mathrm{HCC}$, implying that they are therapeutic targets for HCC treatments [10, 18]. Various studies have also reported the overexpression of histone methyltransferases/demethylases in HCC, such as SMYD2/3 [19], SUV39H1/2, G9a and Dot1L [20]. However, analyses for prognostic markers of HCC have been insufficient for these histone methyltransferases/ demethylases. To elucidate whether these enzymes may be prognostic biomarkers in $\mathrm{HCC}$, we examined the prognostic value of 20 histone methyltransferases and cancer outcomes using the gene expression data from the NCI patient cohort and selected PRMT1, which was most strongly associated with HCC patient overall survival (Supplementary Table 2 and Supplementary Figure 1).

\section{Predictive value of PRMT1 in HCC patient prognosis}

To determine whether PRMT1 has a prognostic value in $\mathrm{HCC}$, we analyzed gene expression data from 441 samples obtained from three independent HCC patient cohorts. We divided the patients from the NCI cohort into two groups using a PRMT1 expression threshold obtained from Receiver operating characteristic (ROC) analysis, as the frequency of overall survival was significantly higher in the high-PRMT1 group compared with the lowPRMT1 group (log-rank test, $P=0.016$; Figure 3A and 3B). By applying the same procedure to the Korean cohort, a consistent statistical significance for the prediction of overall survival was also obtained (log-rank test, $P=$ 0.024; Figure 3C). When estimating PRMT1 expression in the Fudan cohort, however, we did not find a statistical significance, but instead observed a trend for classifying high-risk HCC patients by PRMT1 expression (Figure 3D),
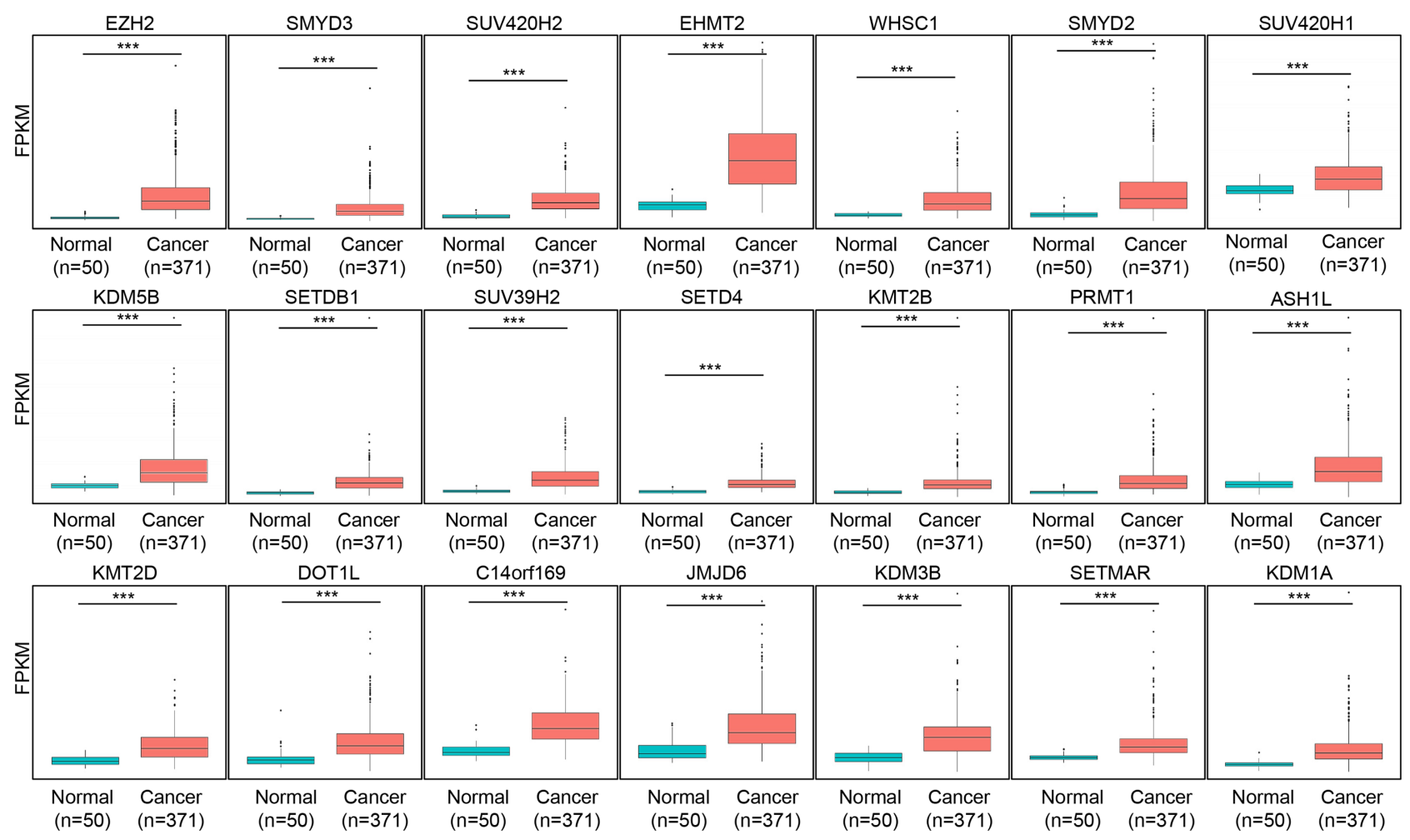

Figure 2: Box plots of the expression of 21 genes were grouped into normal and tumor sets in TCGA liver data set. The $\mathrm{p}$ values were calculated using Student's t-test $\left({ }^{* * *} \mathrm{p}<0.001\right)$. 
indicating the limitations of using a single gene as a diagnostic tool.

\section{The suppression of HCC growth by the knocking down PRMT1 expression in HCC cell lines}

PRMT1 is a protein arginine methyltransferase that catalyzes the methylation of the third arginine in histone H4 after experimental validation. In previous reports, PRMT1 was significantly elevated in several types of cancer and played an important role in cancer progression. PRMT1 expression is also associated with poor prognosis in breast and gastric cancers $[16,21]$, though no study has fully addressed the clinical relevance and function of PRMT1 in HCC.

In HCC patients $(n=371)$, PRMT1 expression levels gradually increased according to the HCC stages and T/N factor (data not shown), implying that PRMT1 expression may be associated with HCC malignancy and proliferation. To investigate the role of PRMT1 in HCC growth, we performed growth analysis after knocking down PRMT1 expression in HCC cell lines. qRT-PCR analysis showed that PRMT1 was clearly suppressed after treatment with siPRMT1 compared with siCont in the SNU182 and 475 cell lines (Figure 4A). In a colony formation assay, the number of HCC cells was significantly reduced after
A

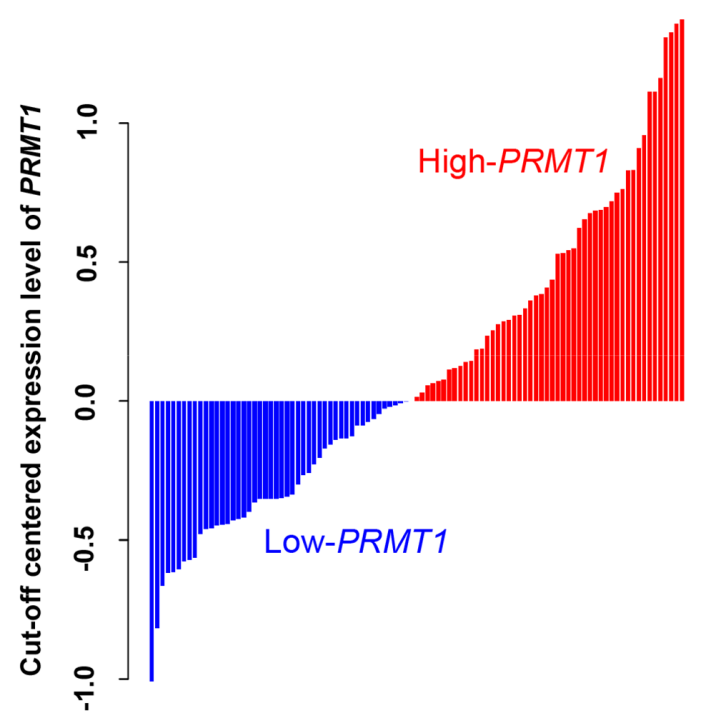

C

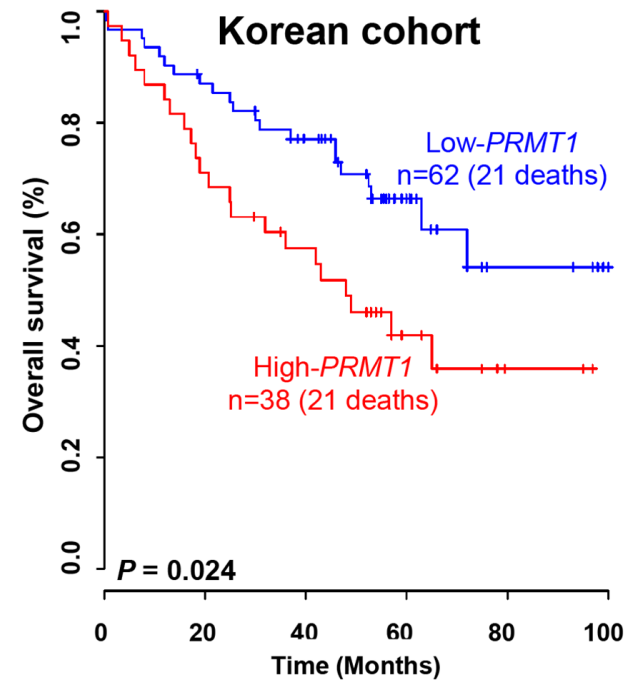

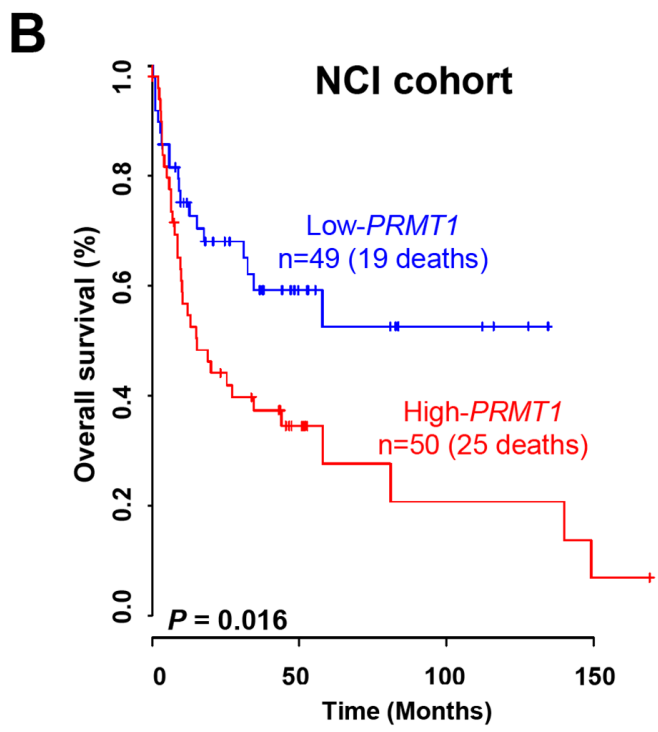

D

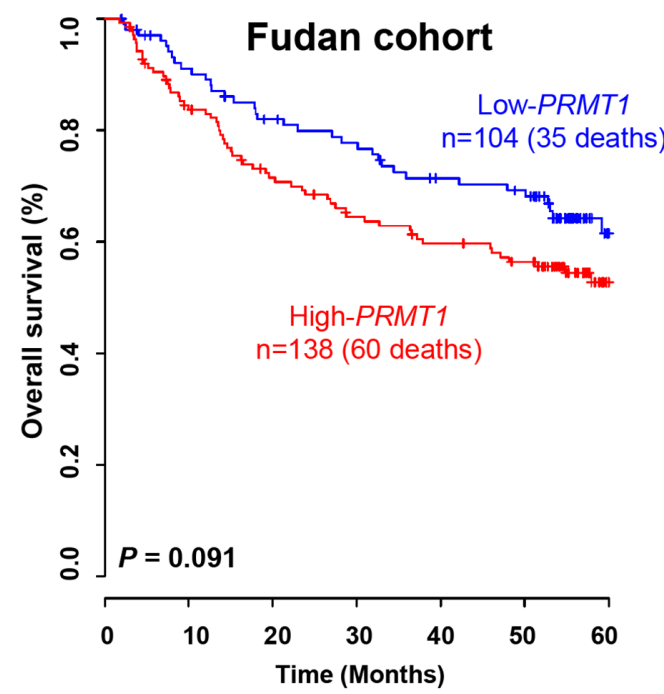

Figure 3: Expression of PRMT1 and prognosis of liver cancer. (A) Expression of PRMT1 in HCC patients from the NCI. Each cutoff value of PRMT1 expression was calculated by ROC analysis in each patient cohort. (B, C, D) Kaplan-Meier curves of overall survival in the (B) NCI, (C) Korean, and (D) Fudan cohorts. 
treatment with PRMT1 siRNAs (Figure 4B and 4C). The use of 3D culture models more closely reflects the tumor compared to 2D culture $[22,23]$. Thus, we performed 3D culture with HCC cell lines using spheroid microplates, which are characterized by ultralow attachment. After PRMT1 knockdown, the formation of spheroids was reduced compared with siCont-treated SNU182 and 475 cells (Figure 4D). FACS analysis showed an induction of cells in Sub-G1 after PRMT1 knockdown (Figure 4E). Taken together, these results suggest that PRMT1 plays an important role in growth and spheroid formation in $\mathrm{HCC}$ cell lines.

\section{Prognostic relevance of $P R M T 1$ and its associated genes}

We sought to identify a gene set that directly correlated with PRMT1 expression to verify its prognostic relevance in HCC and explore biological interactions between PRMT1 and its associated genes. A total of 1,690 genes correlating with PRMT1 expression were selected from the NCI cohort (Pearson correlation test, $|r|>0.4$ and $P<0.001$ ). Based on hierarchical clustering analysis of the expression patterns of these genes, we divided the HCC samples into the following two groups: (1) the highPRMT1 cluster (HPC) and (2) the low-PRMT1 cluster (LPC) (Figure 5A). The overall survival rate of the HPC patients was significantly lower than the LPC patients (log-rank test, $P=0.01$; Figure $5 \mathrm{~B}$ ). To validate this finding in the NCI cohort, we also used gene expression data from an independent cohort of Korean patients with HCC. Using the same procedure employed with the NCI cohort, the patients in the Korean cohort were divided into two groups (HPC and LPC) by hierarchical cluster analysis using 1,672 genes that overlapped with the 1,690 genes derived from the NCI cohort, and the
A

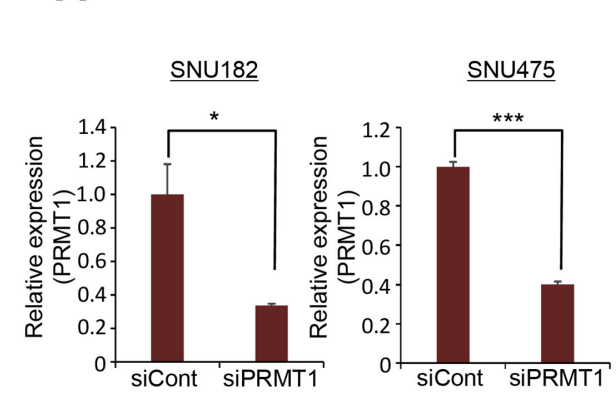

D

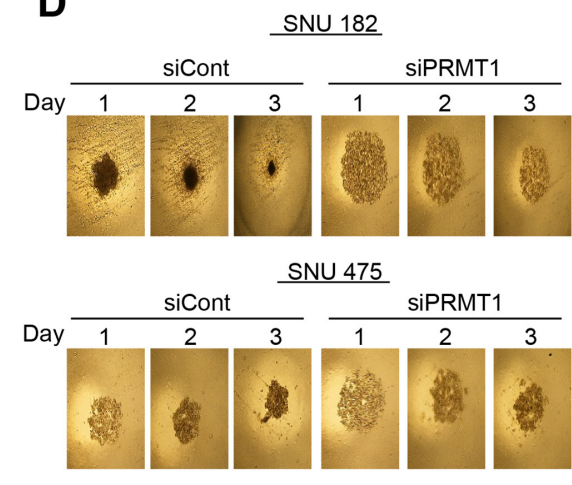

B

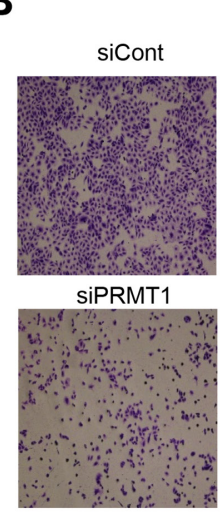

C

SNU182

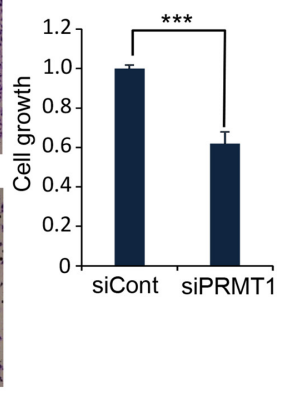

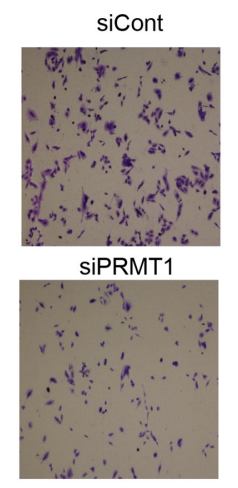

$\underline{\text { SNU475 }}$

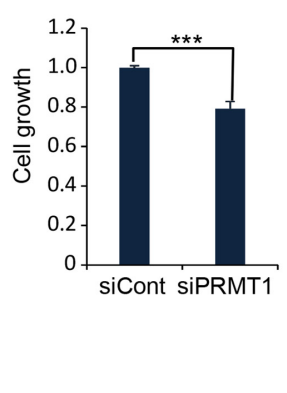

E

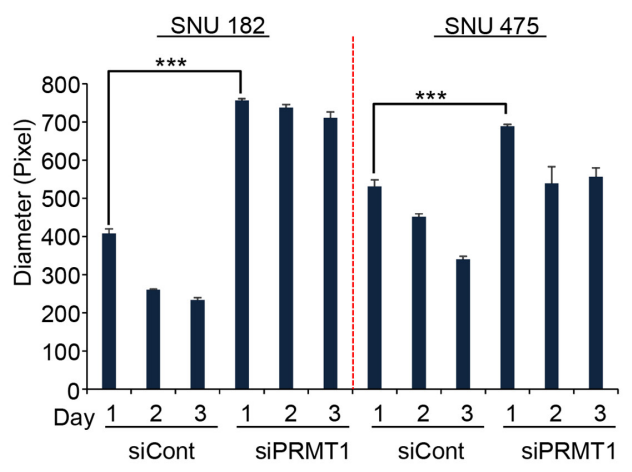

Figure 4: PRMT1 regulated cell growth and spheroid formation in HCC. (A) Knockdown of PRMT1 with siRNA. qPCR analysis after treatment with siPRMT1 and siCont as a negative control for $72 \mathrm{~h}$. The $\mathrm{p}$ values were calculated using Student's t-test $\left({ }^{* * *} \mathrm{p}<0.001,{ }^{*} \mathrm{p}<0.05\right)$. (B and $\left.\mathbf{C}\right)$ Colony formation analysis. After knockdown of PRMT1 for $72 \mathrm{~h}$, cell fixation with $100 \%$ ethanol and cell staining with crystal violet was performed (left), and the quantification of cell number was measured with a microplate reader after disassociating the colony with extraction solution ( $0.9 \%$ trisodium citrate dihydrate, $0.02 \mathrm{ml} / 1 \mathrm{HCl}, 50 \%$ ethanol). (D) Spheroid formation. Cells with siPRMT and siCont were loaded onto spheroid plates and incubated for $72 \mathrm{~h}$. The cells were photographed under a microscope each day (left), and the size of spheroid was measured by image J program (right) $\left({ }^{* * *} \mathrm{p}<0.001\right)$. (E) Cell cycle analysis. After knockdown of PRMT1, cell cycle analysis was performed with MUSE after staining with a cell cycle staining solution. 
overall survival of each group was estimated. KaplanMeier analysis revealed that the PRMT1 gene set was a significant predictor of HCC overall survival in the Korean cohort ( $P=0.03$ by log-rank test; Figure $5 C$ ). When applying the same clustering algorithms and Kaplan-Meier analyses to the Fudan cohort with 1,022 overlapping genes, the survival rate of the high-risk patients was significantly lower than the low-risk patients (log-rank test, $P=0.003$; Figure 5D). Moreover, the recurrence rate of the HPC subgroup classified by PRMT1 and its associated genes was significantly higher than the LPC subgroup (log-rank test, $P=0.05$; Figure 5E).

To determine the independent utility of the newly identified signature based on PRMT1 expression, we combined the clinical data from two test cohorts and applied Cox regression analyses to our signature and known clinicopathological risk factors. In the univariate analysis, significant prognostic indicators of HCC overall survival included the PRMT1 signature, alpha-fetoprotein (AFP), tumor size, and Barcelona Clinic Liver Cancer (BCLC) stage (Supplementary Table 3). When the multivariate test was performed on the pooled cohort, the PRMT1 signature still retained its statistical significance for overall survival of HCC even after applying a variable selection procedure $(\mathrm{HR}=1.612,95 \% \mathrm{CI}=1.127-$ 2.306, $P=0.009$; Supplementary Table 3), demonstrating prognostic relevance of the PRMT1 signature as an independent risk factor for HCC.
A

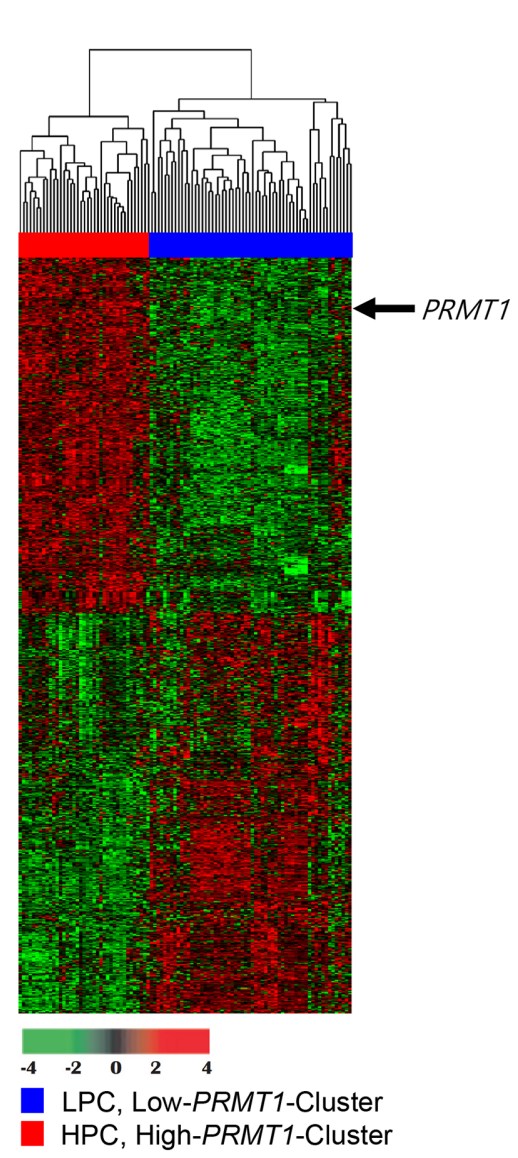

B

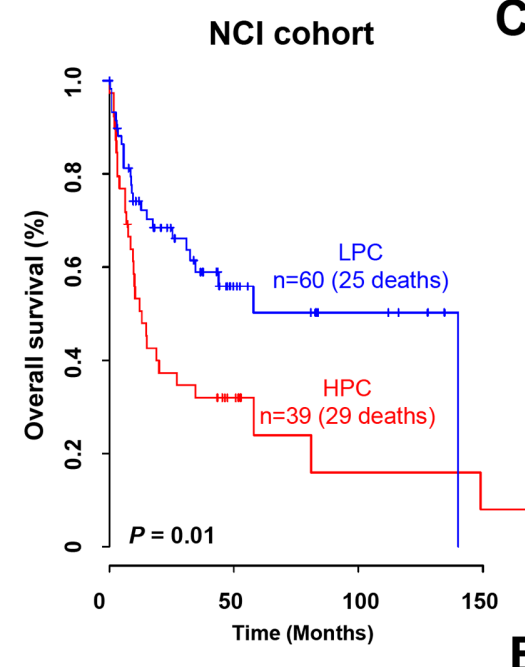

Fudan cohort

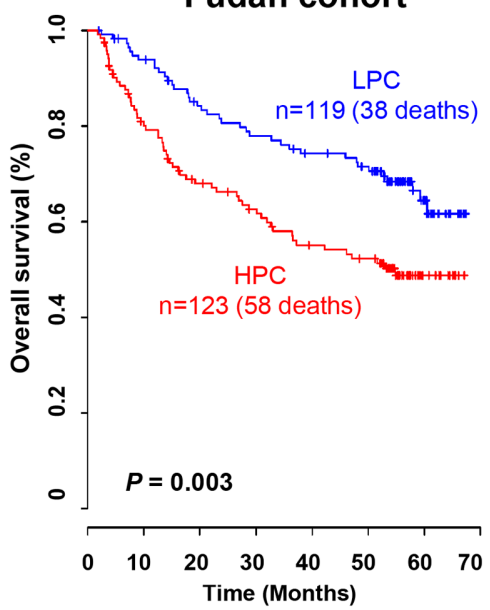

C

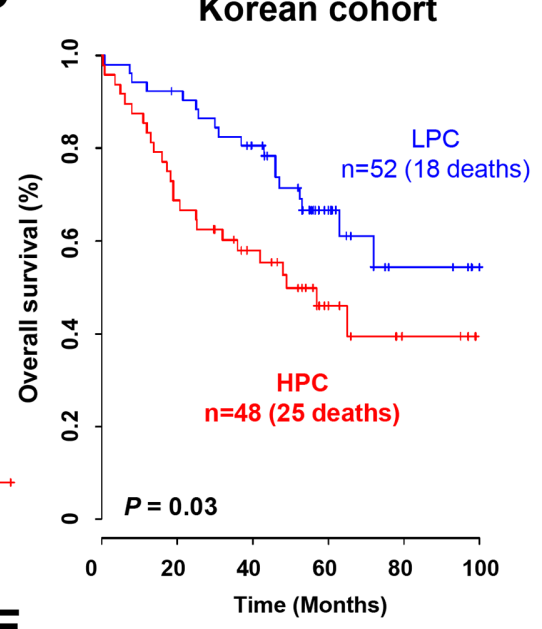

E

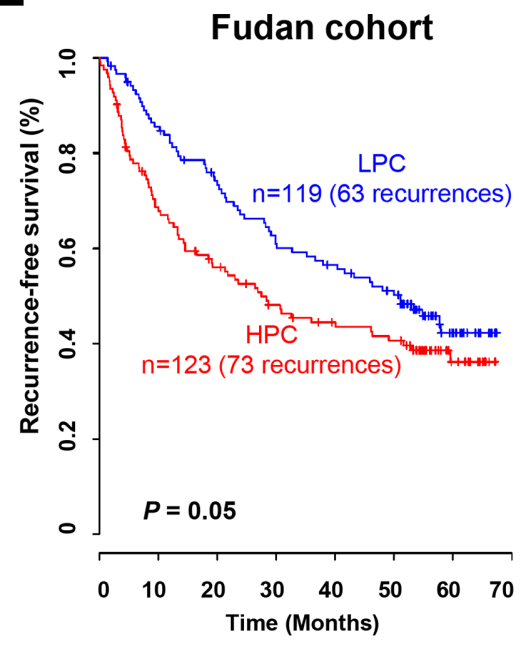

Figure 5: Gene expression pattern of the PRMT1 signature and prognosis of two clusters in the NCI, Korean and Fudan cohorts. (A) Gene expression patterns of PRMT1 and its associated genes in the NCI cohort $(\mathrm{n}=99)$. A total of 1,690 genes with expression patterns that highly correlated with $P R M T 1$ were selected for a cluster analysis (Pearson correlation test, $|r|>0.4$ and $P<$ 0.001). The patients were divided into two groups: a low-PRMT1 cluster (LPC) and high- $P R M T 1$ cluster (HPC). (B-D) Kaplan-Meier plot depicting overall survival in the NCI cohort. The survival rate of the HPC patients was significantly increased compared with LPC patients $(P=0.01$ by log-rank test). Overall survival rates of the two patient groups classified by the $P R M T 1$ signature were validated (C) in the Korean cohort $(\mathrm{n}=100)$ and $(\mathrm{D})$ in the Fudan cohort $(\mathrm{n}=242)$. (E) The PRMT1 signature also showed a predictive value for recurrencefree survival in the Fudan cohort. 


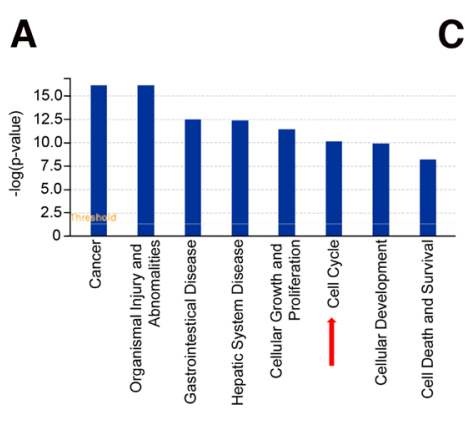

B
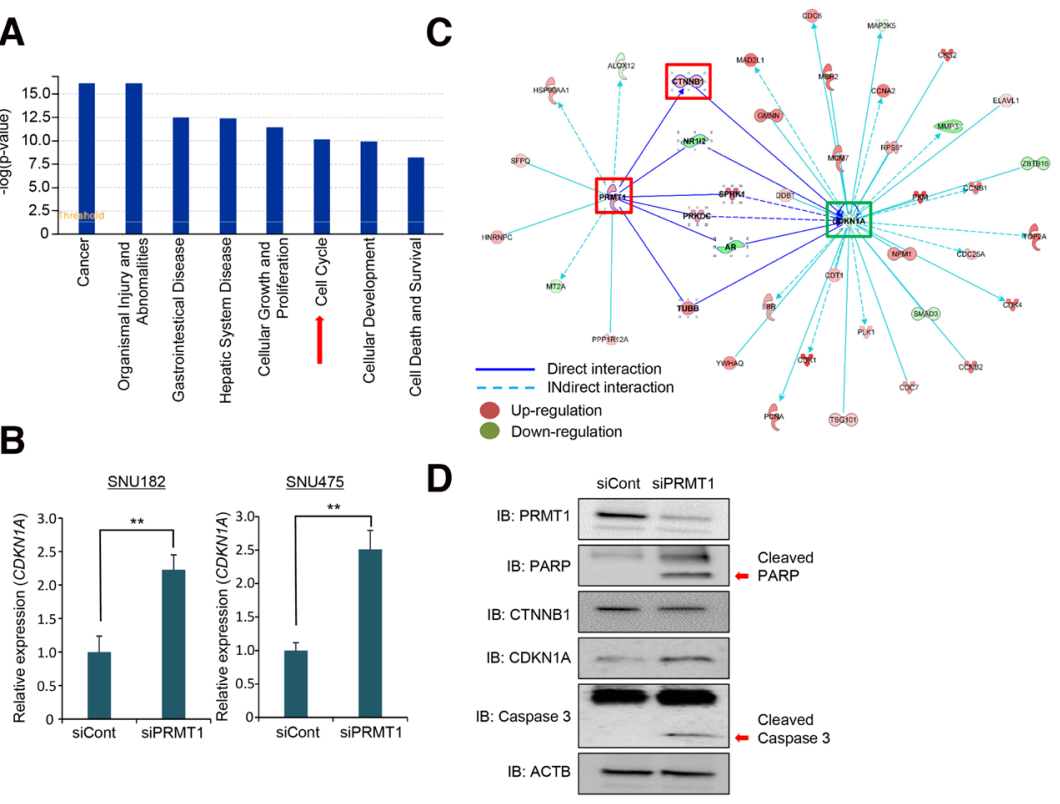

E
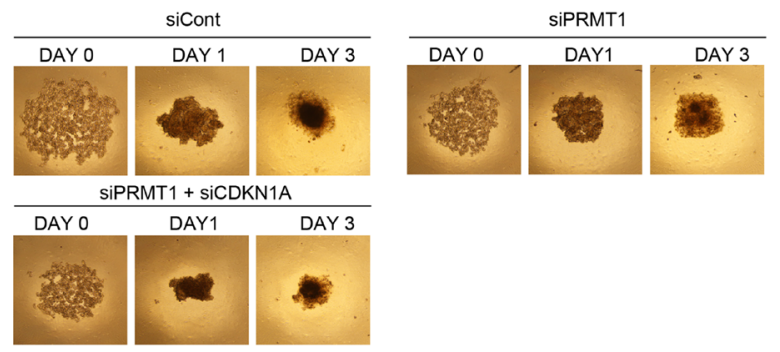

$\mathbf{F}$
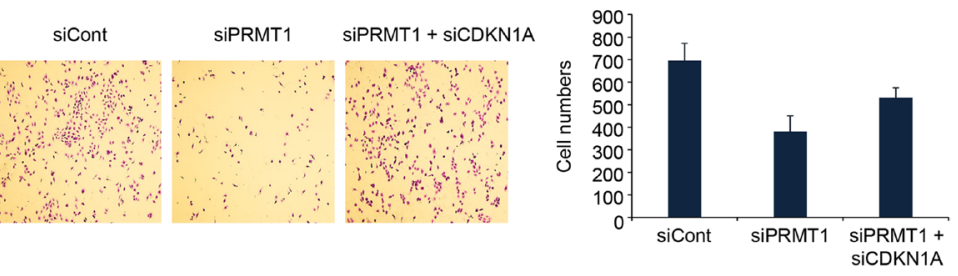

G

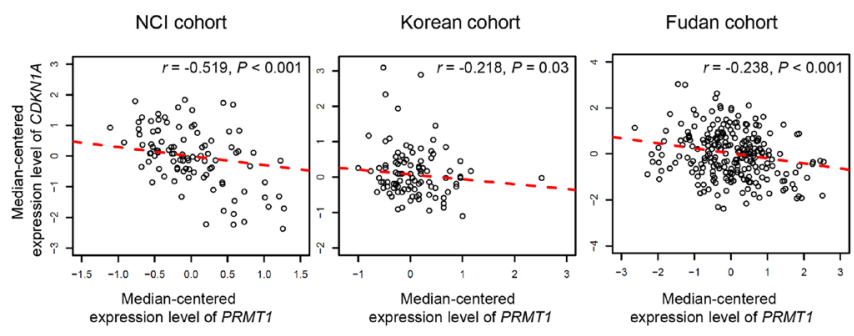

Figure 6: Reverse-correlation between PRMT1 and CDKN1A in HCC clinical cohorts. (A) Canonical pathway analysis. Histograms represent the canonical pathways. (B) Upregulation of CDKN1A by knockdown of PRMT1. qPCR analysis after treatment with siPRMT1 and siCont as a negative control for $72 \mathrm{~h}$. The p values were calculated using the Student's t-test ( $\left.{ }^{* *} \mathrm{p}<0.01\right)$. (C) Geneto-gene networks of PRMTI and CDKNIA associated with hepatocellular carcinoma (HCC) prognosis. Up- and downregulated genes in the high-PRMT1 cluster (HPC) group are indicated in red and green, respectively. The intensity of the color is indicative of the degree of over- or under-expression. Each line and arrow represents functional and physical interactions between the genes and the direction of regulation reported in the literature. (D) Western blot analysis of PRMT1, PARP1, Caspase 3, CTNNB1 and CDKN1A. ACTB was used as an internal control. (E) Spheroid formation. Cells treated with siPRMT, siCont and siPRMT1/siCDKN1A were loaded onto spheroid plates and incubated for $72 \mathrm{~h}$. The cells were photographed under a microscope each day. (F) Colony formation analysis. After treatment with siPRMT, siCont and siPRMT1/siCDKN1A for $72 \mathrm{~h}$, the cells were fixed with $100 \%$ ethanol, stained with crystal violet (left), and the cell number was determined with the ImageJ program (right). (G) Scatter plots of PRMTI and CDKNIA in the NCI, Korean and Fudan cohorts. Each red dotted line indicates a linear regression line of the expression of PRMT1 and CDKNIA. $P$ values and correlation coefficients $(r)$ between two genes were obtained by the Pearson correlation method. 


\section{Active regulators in the prognostic gene set associated with PRMT1}

To explore the biological characteristics and interactions among PRMT1-associated genes, a gene set enrichment test was performed using the IPA software on the 1,690 genes. The analysis showed that genes involved in cancer, cell growth and proliferation, and tissue development were significantly enriched. Among the genes associated with PRMT1, a significant number were identified as being involved in the cell cycle, indicating that altered biological processes associated with the cell cycle might be markedly responsible for poor prognosis in HCC (Figure 6A). Among PRMT1-associated genes, we performed a candidate approach to identify cell cyclerelated genes and selected $C D K N 1 A$. CDKN1A, which is a cyclin dependent kinase inhibitor, drives cell cycle arrest in response to various environmental stimuli [24]. PRMT1 knockdown induced the upregulation of CDKN1A transcription compared with siCont-treated HCC cell lines (Figure 6B). In an analysis of PRMT1 and CDKN1A geneto-gene networks, upregulated $P R M T 1$ enhanced $C T N N B 1$ expression, and upregulated $C T N N B 1$ induced by PRMT1 reduced $C D K N 1 A$ expression (Figure $6 \mathrm{C}$ ). To confirm this result at the protein level, we performed Western blot analysis after PRMT1 knockdown and found that PRMT1 knockdown downregulated CTNNB1, induced CDKN1A, and cleaved PARP1 and Caspase 3 in the SNU182 cell line, implying that downregulated CTNNB1 induced by PRMT1 promoted CDKN1A expression and cell apoptosis (Figure 6D). Additionally, to assess whether CDKN1A is a final effector protein in this pathway, rescue experiments were performed after co-transfection with siPRMT1 and siCDKN1A. In 3D culture analysis, the cells treated with siPRMT1 had difficulty forming spheroids compared with siCont-treated cells. However, co-transfection with siPRMT1 and siCDKN1A induced spheroid formation compared to siPRMT1 treatment only (Figure 6E). Additionally, in the cell growth assay, we observed the recovery of cell growth after co-treatment with both siPRMT1 and siCDKN1A (Figure 6F). Thus, we believe that CDKN1A expression may be involved downstream of PRMT1 and that regulation of CDKN1A by PRMT1 plays an important factor for tumor growth and formation in HCC.

Finally, to validate the findings from the gene network analysis in clinical samples, we examined the rate of reverse correlation using clinical cohorts. Figure $6 \mathrm{G}$ shows the statistically significant reverse correlations [Pearson correlation tests; $\mathrm{r}=-0.519, P<0.001$ (NCI); $\mathrm{r}=-0.218, P=0.03$ (Korean); $\mathrm{r}=-0.238, P<0.001$ (Fudan)] between PRMT1 and CDKN1A. These results suggest that overexpressed PRMT1 in HCC can downregulate CDKN1A expression levels and subsequently may promote the proliferation and growth of cancer cells in response to various stimuli.

\section{DISCUSSION}

RNA-seq is a useful tool for analyzing the whole transcriptomes of cancer cells at the levels of genes and exons involving novel splicing variants [25]. In melanoma, for the first time, novel gene fusions and read-through transcripts were identified using RNA-seq to discover cancer targets [26]. In this study, we established a screening pipeline based on RNA-seq to identify overexpressed histone methyltransferases as prognostic markers in HCC. The advantages of RNA-seq include its high sensitivity and quantification of gene expression levels. In the screening pipeline, we used public RNA-seq data from 50 normal livers and $371 \mathrm{HCC}$ tissues derived from the TCGA portal and analyzed the expression levels with an in silico panel of histone methyltransferases/ demethylases (Figure 1). We selected 21 of these histone methyltransferases/demethylases with a greater than 2 -fold change in expression in HCC compared with normal tissues. Among them, we assessed the possibility of these genes being prognostic biomarkers in HCC using the NCI cohort. Several prognostic systems for estimating tumor stage have been developed, including the CLIP score, Okuda staging, and BCLC. Okuda staging and $\mathrm{BCLC}$ are based on tumor size and serum albumin and bilirubin levels, and the CLIP score predicts HCC based on the Child-Pugh stage, AFP level and tumor morphology [27]. However, there are limits to predicting the stage of HCC patients with the currently used systems. Therefore, to better evaluate HCC patients, novel prognostic biomarkers and assessment systems have been continuously required. Here, using our screening pipeline, we identified PRMT1 as a novel prognostic biomarker for the prediction of a response to therapeutic intervention in HCC (Figure 3).

PRMT can be classified according to the position of methylation, including three types (I, II and III). Of note, type 1 PRMTs $(1,3,4,6$ and 8$)$ have been thought of as attractive therapeutic targets for developing specific inhibitors because of their overexpression in various types of cancers [28]. Among them, the overexpression of PRMT1 has been observed in lung, breast, bladder, colon, and prostate cancer and leukemia [29]. Here, we also demonstrated the overexpression of PRMT1 in HCC clinical samples and that the PRMT1 knockdown inhibited growth and spheroid formation in HCC cell lines, implying that PRMT1 inhibitors may be an effective therapeutic method in HCC treatment. PRMT1 has three distinct isoforms, with PRMT1 v1 being the major isoform [30]. In colon cancer, the levels of the PRMT1 v1 isoform are clearly associated with clinical and pathological variables [31]. Additionally, in breast cancer, patients with low expression of PRMT1 v1 display longer disease-free survival [16]. However, in gastric cancer, low expression of PRMT1 is associated with poor prognosis [21]. In $\mathrm{HCC}$, as with colon and breast cancer, high expression 
of PRMT1 was significantly related to a poor prognosis, which was confirmed with the Korean cohort. Moreover, the PRMT1-associated gene set clearly reflected the poor prognosis in the NCI, Korean and Fudan cohorts. Prognostic markers can help lead to clinical treatments by identifying patients with similar cases. Moreover, prognostic markers can also help compare differences between clinical trials and patient groups in accordance with risk factors [32]. Therefore, the novel prognostic marker PRMT1 in HCC may help identify subgroups of patients and be used to design new treatments for HCC treatment.

Recently, several papers have demonstrated that RAB27B regulates cell cycle progression through the $\mathrm{PI} 3 \mathrm{~K} / \mathrm{AKT} / \mathrm{CDKN} 1 \mathrm{~A}$ pathway and by modulating cell growth in HCC cell lines, suggesting that upregulation of CDKN1A contributes to HCC cell growth. Moreover, MDIG expression levels could effect CDKN1A expression through H3K9 tri-methylation and the induction of CDKN1A expression by MDIG knockdown associated with HCC growth [33, 34]. And Wang's group also demonstrated that overexpression of miR-95-3p regulated expression of P21 for hepatocarcinogenesis [35]. Taken together, we could suggest that the regulation of CDKN1A in HCC by several effector genes including PRMT1 can influence tumor growth.

In summary, our screening pipeline system was easily applied to identify a novel prognostic marker in several types of cancer. PRMT1 is a novel prognostic marker and a potentially therapeutic target that may play an important role in HCC treatment.

\section{MATERIALS AND METHODS}

\section{Datasets of HCC patients including TCGA data}

A total of four cohorts of patients with HCC including the TCGA data were used for this study. The mRNA expression (RNA-Seq) data was obtained from TCGA data portal (http://cancergenome.nih.gov) for liver related 421 samples (50 normal samples and 371 tumor samples). We downloaded RNA-Seq quantification data (HTSeq-FPKM) and calculated mean value for the expression levels of each gene across samples. These mean values represent expression each gene about normal and tumor. For verifying a prognostic value of PRMT1, we also obtained gene expression datasets of HCC patients from the National Cancer Institute (GSE1898, n = 99, the NCI cohort), Fudan University Liver Cancer Institute (GSE14520, $\mathrm{n}=242$, the Fudan cohort), and Seoul and Chonbuk National University of Korea (GSE16757, n $=100$, the Korean cohort), which are available from the NCBI's GEO database. The baseline characteristics of the three cohorts of HCC patients for verifying prognostic relevance of PRMT1 were described in Supplementary Table 1.

\section{Cell culture}

The human liver cancer cell lines SNU 182 and 475 were purchased from the Korean Cell Line Bank (Seoul, South Korea) and were cultured in RPMI supplemented with $10 \%$ fetal bovine serum (FBS) and 1\% penicillin/ streptomycin in a humidified atmosphere of $5 \% \mathrm{CO}_{2}$ at $37^{\circ} \mathrm{C}$ according to the manufacturer's instructions [36].

\section{siRNA transfection}

Control, PRMT1 and CDKN1A siRNA duplexes were purchased from ST Pharm (Seoul, South Korea) and Bioneer (Seoul, South Korea). siCont (negative control siRNA) was used for the control treatment. siRNA sequences are described in Supplementary Table 4. In total, $100 \mathrm{nM}$ siRNAs were transfected into cancer cell lines using RNAiMAX (Invitrogen, Carlsbad, CA) for $48 \mathrm{~h}$.

\section{Western blot analysis}

Western blotting was performed according to the manufacturer's instructions [36]. The cells were washed once with $\mathrm{PBS}$ and then lysed in cold lysis buffer (50 mM Tris- $\mathrm{HCl}, \mathrm{pH} 7.4,150 \mathrm{mM} \mathrm{NaCl}, 1 \%$ Triton $\mathrm{X}-100,0.1 \%$ SDS, $1 \mathrm{mM}$ EDTA, $1 \mathrm{mM} \mathrm{Na} \mathrm{VO}_{4}, 1 \mathrm{mM}$ $\mathrm{NaF}$, and $1 \times$ protease inhibitor cocktail). Cell lysates were centrifuged at $14,000 \times \mathrm{g}$ for $15 \mathrm{~min}$ at $4^{\circ} \mathrm{C}$ and then boiled in $5 \times$ sample buffer. The protein samples were subjected to Western blotting with the indicated antibodies at a 1:500 dilution ratio. The samples were stained with the anti-PRMT1 (B-2), ACTB (SC-4778) and B-catenin (SC-7963) antibodies from Santa Cruz (Santa Cruz, CA, USA) and the anti-PARP (\#9542S) and Caspase 3 (\#9665S) antibodies from Cell Signaling Technology (MA, USA).

\section{Semi-quantitative reverse transcription PCR and quantitative real-time PCR}

Total RNA was isolated from the indicated cell lines using a Qiagen RNeasy Mini Kit according to the manufacturer's instructions [37-39]. RNA aliquots of $1 \mu \mathrm{g}$ were then reverse transcribed using the iScript ${ }^{\mathrm{TM}}$ cDNA synthesis kit (Bio-Rad, Hercules, CA), according to the standard protocols. For semi-quantitative RTPCR, cDNA was used as a template for PCR using AccuPower® ProFi Taq PCR PreMix (Bioneer, Daejeon, South Korea). For quantitative RT-PCR, PCR reactions were performed using the CFX96 Real-Time System (Bio-Rad) following the manufacturer's instructions. PCR primers were as follows: PRMT1 (Forward primer 5'-GGGCTACTGCCTCTTCTACGAGTC-3', Reverse primer 5'-GTCTTTGTACTGCCGGT CCTCGTAG-3'), CDKN1A (Forward primer 
5'-GGGATGTCCGT CAGAACCCA -3', Reverse primer 5'-CACCCTCCAGTGGTGTCTCG -3').

\section{Spheroid formation}

To perform spheroid culture of HCC cell lines, ultralow attachment microplates were used (Corning, Cat. 4515). After knockdown of PRMT1, a concentration of 2 $\times 10^{4}$ cells was loaded onto spheroid culture plates for 3 days then observed using microscopy.

\section{FACS analysis}

After knockdown of PRMT1 for 3 days, the collected cells were washed with PBS and fixed with 70\% cold ethanol for $3 \mathrm{~h}$ at -20 degrees. After washing with PBS, cell cycle solution (Millipore, MCH100106) was added and the cells were analyzed using a MUSE cell analyzer.

\section{Statistical analysis}

To classify patients into two groups by PRMT1 expression in the HCC gene expression data, we obtained an optimal cutoff of PRMT1 expression from ROC analysis, in which the best cutoff was determined by the expression with the highest sensitivity and specificity. The Kaplan-Meier method was used to calculate the time to death or recurrence, and differences between the times were assessed using log-rank statistics. Pearson correlation coefficients were calculated to evaluate the association between PRMT1 and its correlated genes. A hierarchical clustering algorithm, using the centered correlation coefficient as the measure of similarity and complete linkage clustering, was applied. The prognostic association between PRMT1 signature and clinicopathological risk factors was assessed using multivariate Cox proportional hazard models. A backward-forward step procedure (function step, $\mathrm{R}$ package stats) was applied to optimize the multivariate model with the most informative variables.

A gene set enrichment analysis was performed to identify the most significant gene sets associated with the disease process, molecular and cellular functions, and physiological and development conditions. The significance of over-represented gene sets was estimated by the Fisher exact test. To explore the relationships between the genes in the gene set associated with PRMT1, we performed an upstream regulator analysis that searched known targets of each regulator in the data set and compared their direction of change to the expected change based on previously published literature. Gene set enrichment and upstream regulator analyses were performed using the Ingenuity Pathway Analysis (IPA, Ingenuity Systems, www. ingenuity.com).

\section{ACKNOWLEDGMENTS AND FUNDING}

This study was supported by the Basic Science Research Program of the National Research Foundation of Korea (NRF) funded by the Ministry of Education, Science and Technology (NRF-2017R1A2B4003757, 2016M3A9C4953144) and by the KRIBB Research Initiative Program.

\section{CONFLICTS OF INTEREST}

The authors declare no conflicts of interest.

\section{REFERENCES}

1. El-Serag HB, Mason AC. Rising incidence of hepatocellular carcinoma in the United States. N Engl J Med. 1999; 340:745-750.

2. Llovet JM, Burroughs A, Bruix J. Hepatocellular carcinoma. Lancet. 2003; 362:1907-1917.

3. Llovet JM, Ricci S, Mazzaferro V, Hilgard P, Gane E, Blanc JF, de Oliveira AC, Santoro A, Raoul JL, Forner A, Schwartz M, Porta C, Zeuzem S, et al. Sorafenib in advanced hepatocellular carcinoma. N Engl J Med. 2008; 359:378-390.

4. Chen J, Jin R, Zhao J, Liu J, Ying H, Yan H, Zhou S, Liang Y, Huang D, Liang X, Yu H, Lin H, Cai X. Potential molecular, cellular and microenvironmental mechanism of sorafenib resistance in hepatocellular carcinoma. Cancer Lett. 2015; 367:1-11.

5. Hardy T, Mann DA. Epigenetics in liver disease: from biology to therapeutics. Gut. 2016.

6. Herceg Z, Paliwal A. Epigenetic mechanisms in hepatocellular carcinoma: how environmental factors influence the epigenome. Mutat Res. 2011; 727:55-61.

7. Ozen C, Yildiz G, Dagcan AT, Cevik D, Ors A, Keles U, Topel H, Ozturk M. Genetics and epigenetics of liver cancer. N Biotechnol. 2013; 30:381-384.

8. Cai MY, Hou JH, Rao HL, Luo RZ, Li M, Pei XQ, Lin MC, Guan XY, Kung HF, Zeng YX, Xie D. High expression of H3K27me3 in human hepatocellular carcinomas correlates closely with vascular invasion and predicts worse prognosis in patients. Mol Med. 2011; 17:12-20.

9. He C, Xu J, Zhang J, Xie D, Ye H, Xiao Z, Cai M, Xu K, Zeng Y, Li H, Wang J. High expression of trimethylated histone $\mathrm{H} 3$ lysine 4 is associated with poor prognosis in hepatocellular carcinoma. Hum Pathol. 2012; 43:1425-1435.

10. Cai MY, Tong ZT, Zheng F, Liao YJ, Wang Y, Rao HL, Chen YC, Wu QL, Liu YH, Guan XY, Lin MC, Zeng YX, Kung $\mathrm{HF}$, Xie D. EZH2 protein: a promising immunomarker for the detection of hepatocellular carcinomas in liver needle biopsies. Gut. 2011; 60:967-976. 
11. Sasaki M, Ikeda H, Itatsu K, Yamaguchi J, Sawada S, Minato H, Ohta T, Nakanuma Y. The overexpression of polycomb group proteins Bmil and EZH2 is associated with the progression and aggressive biological behavior of hepatocellular carcinoma. Lab Invest. 2008; 88:873-882.

12. Zhao ZK, Yu HF, Wang DR, Dong P, Chen L, Wu WG, Ding WJ, Liu YB. Overexpression of lysine specific demethylase 1 predicts worse prognosis in primary hepatocellular carcinoma patients. World J Gastroenterol. 2012; 18:6651-6656.

13. An W, Kim J, Roeder RG. Ordered cooperative functions of PRMT1, p300, and CARM1 in transcriptional activation by p53. Cell. 2004; 117:735-748.

14. Yoshimatsu M, Toyokawa G, Hayami S, Unoki M, Tsunoda T, Field HI, Kelly JD, Neal DE, Maehara Y, Ponder BA, Nakamura Y, Hamamoto R. Dysregulation of PRMT1 and PRMT6, Type I arginine methyltransferases, is involved in various types of human cancers. Int J Cancer. 2011; 128:562-573.

15. Cheung N, Fung TK, Zeisig BB, Holmes K, Rane JK, Mowen KA, Finn MG, Lenhard B, Chan LC, So CW. Targeting aberrant epigenetic networks mediated by PRMT1 and KDM4C in acute myeloid leukemia. Cancer Cell. 2016; 29:32-48.

16. Mathioudaki K, Scorilas A, Ardavanis A, Lymberi P, Tsiambas E, Devetzi M, Apostolaki A, Talieri M. Clinical evaluation of PRMT1 gene expression in breast cancer. Tumour Biol. 2011; 32:575-582.

17. Li B, Liu L, Li X, Wu L. miR-503 suppresses metastasis of hepatocellular carcinoma cell by targeting PRMT1. Biochem Biophys Res Commun. 2015; 464:982-987.

18. Zhao ZK, Dong P, Gu J, Chen L, Zhuang M, Lu WJ, Wang DR, Liu YB. Overexpression of LSD1 in hepatocellular carcinoma: a latent target for the diagnosis and therapy of hepatoma. Tumour Biol. 2013; 34:173-180.

19. Hamamoto R, Nakamura Y. Dysregulation of protein methyltransferases in human cancer: an emerging target class for anticancer therapy. Cancer Sci. 2016; 107:377-384.

20. Hung SY, Lin HH, Yeh KT, Chang JG. Histone-modifying genes as biomarkers in hepatocellular carcinoma. Int J Clin Exp Pathol. 2014; 7:2496-2507.

21. Altan B, Yokobori T, Ide M, Mochiki E, Toyomasu Y, Kogure N, Kimura A, Hara K, Bai T, Bao P, Suzuki M, Ogata K, Asao T, et al. Nuclear PRMT1 expression is associated with poor prognosis and chemosensitivity in gastric cancer patients. Gastric Cancer. 2016; 19:789-797.

22. Lancaster MA, Knoblich JA. Organogenesis in a dish: modeling development and disease using organoid technologies. Science. 2014; 345:1247125.

23. Ramachandran SD, Schirmer K, Munst B, Heinz S, Ghafoory S, Wolfl S, Simon-Keller K, Marx A, Oie CI, Ebert MP, Walles H, Braspenning J, Breitkopf-Heinlein $\mathrm{K}$. In vitro generation of functional liver organoid-like structures using adult human cells. PLoS One. 2015; 10:e0139345.

24. Abbas T, Dutta A. p21 in cancer: intricate networks and multiple activities. Nat Rev Cancer. 2009; 9:400-414.

25. Wang Z, Gerstein M, Snyder M. RNA-Seq: a revolutionary tool for transcriptomics. Nat Rev Genet. 2009; 10:57-63.

26. Berger MF, Levin JZ, Vijayendran K, Sivachenko A, Adiconis X, Maguire J, Johnson LA, Robinson J, Verhaak RG, Sougnez C, Onofrio RC, Ziaugra L, Cibulskis K, et al. Integrative analysis of the melanoma transcriptome. Genome Res. 2010; 20:413-427.

27. Greten TF, Papendorf F, Bleck JS, Kirchhoff T, Wohlberedt T, Kubicka S, Klempnauer J, Galanski M, Manns MP. Survival rate in patients with hepatocellular carcinoma: a retrospective analysis of 389 patients. Br J Cancer. 2005; 92:1862-1868.

28. Hu H, Qian K, Ho MC, Zheng YG. Small molecule inhibitors of protein arginine methyltransferases. Expert Opin Investig Drugs. 2016; 25:335-358.

29. Yang Y, Bedford MT. Protein arginine methyltransferases and cancer. Nat Rev Cancer. 2013; 13:37-50.

30. Scorilas A, Black MH, Talieri M, Diamandis EP. Genomic organization, physical mapping, and expression analysis of the human protein arginine methyltransferase 1 gene. Biochem Biophys Res Commun. 2000; 278:349-359.

31. Mathioudaki K, Papadokostopoulou A, Scorilas A, Xynopoulos D, Agnanti N, Talieri M. The PRMT1 gene expression pattern in colon cancer. Br J Cancer. 2008; 99:2094-2099.

32. Winter JN. Prognostic markers in diffuse large B-cell lymphoma: keys to the underlying biology. Curr Hematol Malig Rep. 2007; 2:235-241.

33. Huo Q, Ge C, Tian H, Sun J, Cui M, Li H, Zhao F, Chen T, Xie H, Cui Y, Yao M, Li J. Dysfunction of IKZF1/MYC/ MDIG axis contributes to liver cancer progression through regulating H3K9me3/p21 activity. Cell Death Dis. 2017; 8:e2766.

34. Yang X, Ye X, Sun L, Gao F, Li Y, Ji X, Wang X, Feng Y, Wang X. Downregulation of serum RAB27B confers improved prognosis and is associated with hepatocellular carcinoma progression through PI3K-AKT-P21 signaling. Oncotarget. 2017; 8:61118-61132. https://doi.org/10.18632/ oncotarget.18010.

35. Ye J, Yao Y, Song Q, Li S, Hu Z, Yu Y, Hu C, Da X, Li $\mathrm{H}$, Chen Q, Wang QK. Up-regulation of miR-95-3p in hepatocellular carcinoma promotes tumorigenesis by targeting p21 expression. Sci Rep. 2016; 6:34034.

36. Lee JH, Cho HS, Lee JJ, Jun SY, Ahn JH, Min JS, Yoon JY, Choi MH, Jeon SJ, Lim JH, Jung CR, Kim DS, Kim HT, et al. Plasma glutamate carboxypeptidase is a negative regulator in liver cancer metastasis. Oncotarget. 2016; 7:79774-79786. https://doi.org/10.18632/oncotarget.12967. 
37. Cho HS, Kang JG, Lee JH, Lee JJ, Jeon SK, Ko JH, Kim DS, Park KH, Kim YS, Kim NS. Direct regulation of E-cadherin by targeted histone methylation of TALE-SET fusion protein in cancer cells. Oncotarget. 2015; 6:2383723844. https://doi.org/10.18632/oncotarget.4340.

38. Son MY, Kim YD, Seol B, Lee MO, Na HJ, Yoo B, Chang JS, Cho YS. Biomarker discovery by modeling Behcet's disease with patient-specific human induced pluripotent stem cells. Stem Cells Dev. 2017; 26:133-145.

39. Son MY, Sim H, Son YS, Jung KB, Lee MO, Oh JH, Chung SK, Jung CR, Kim J. Distinctive genomic signature of neural and intestinal organoids from familial Parkinson's disease patient-derived induced pluripotent stem cells. Neuropathol Appl Neurobiol. 2017. 